\title{
The Diagnostic and Prognostic Value of Presepsin in Egyptian Patients with Sepsis
}

\author{
Fahmy T. Ali ${ }^{1}$, Mohamed A. M. Ali ${ }^{*}$, Mostafa M. Elnakeeb ${ }^{2}$ and Heba N. M. Bendary ${ }^{2}$ \\ ${ }^{1}$ Department of Biochemistry, Faculty of Science, Ain Shams University, 11566 Abbassia, Cairo, Egypt, \\ ${ }^{2}$ El-Maadi Military Hospital, Cairo, Egypt
}

\section{A R T I C L E I N F O}

Article history:

Received 26 November 2015

Accepted 20 December 2015

Keywords:

Sepsis;

Presepsin;

Procalcitonin;

C-reactive protein.

\begin{abstract}
A B S T R A C T
Despite their undoubted helpfulness in diagnosing sepsis, increased blood Creactive protein (CRP) and procalcitonin (PCT) levels have been described in many noninfectious conditions. Furthermore, CRP and PCT have a limited value for risk stratification and outcome prediction. Presepsin is a soluble fragment of the cluster of differentiation 14 (CD14) involved in pathogen recognition by innate immunity. We aimed to investigate the diagnostic and prognostic performance of presepsin in comparison to PCT and CRP in patients presenting with systemic inflammatory response syndrome (SIRS) and suspected sepsis. Seventy-six subjects were enrolled in this study, including 51 patients with SIRS as well as 25 healthy subjects. Plasma presepsin, PCT and CRP levels were serially measured at 5 time points (on admission and at days 1, 3, 7 and 15). Presepsin and PCT yielded similar diagnostic accuracy with area under the receiver operating characteristic (ROC) curves (AUCs) of 0.805 and 0.780 , respectively, $(P=0.755)$, whereas presepsin performed significantly better than CRP for diagnosing sepsis (AUC, 0.805 vs. $0.613 ; P=0.029$ ). PCT showed a trend towards a better diagnostic performance than CRP (AUC, 0.780 vs. 0.613; $P=0.062$ ). PCT and presepsin showed comparable performance for predicting 28day mortality with AUCs of 0.932 and 0.891 , respectively, $(P=0.465)$, whereas PCT and presepsin performed significantly better than CRP (AUC $=0.445$; $P<0.001)$ in predicting 28 -day mortality. In septic patients, presepsin showed the highest concentration on day 3 and tended to decrease on day 7, whereas PCT and CRP showed the highest concentration on day 7 and tended to decrease on day 15 , suggesting that presepsin revealed earlier concentration changes over time when compared to PCT and CRP. Presepsin and PCT could differentiate between septic and non-septic patients with comparable accuracy and both biomarkers showed similar performance for predicting 28-day mortality. Early changes in presepsin concentrations might reflect the appropriateness of the therapeutic modality and could be useful for making effective treatment decisions.
\end{abstract}

\section{Introduction}

Sepsis is a multifactorial and rapidly progressing disease characterized by an excessive inflammatory response to infection that can lead to organ failure and eventual death. Sepsis refers to the presence of an infection accompanied by evidence of a systemic response called the systemic inflammatory response syndrome (SIRS). SIRS is defined as the presence of 2 or more of the following: (1) temperature greater than $38^{\circ} \mathrm{C}$ (fever) or less than $36^{\circ} \mathrm{C}$ (hypothermia); (2) heart rate greater than 90 beats/minute (tachycardia); (3) respiratory rate greater

* Corresponding author.

E-mail address: m_aly_79@yahoo.com greater than 20 breaths/minute (tachypnea); and (4) white blood cells count greater than $12,000 / \mathrm{mm}^{3}$ (leukocytosis) or less than $4,000 / \mathrm{mm}^{3}$ (leukopenia), or greater than $10 \%$ of the neutrophils are immature ${ }^{[1]}$. The yearly incidence of sepsis is 50-95 cases per 100000 , and has been increasing by $9 \%$ each year. This disease accounts for $2 \%$ of hospital admissions; roughly $9 \%$ of patients with sepsis progress to severe sepsis, and $3 \%$ of those with severe sepsis experience septic shock, which accounts for $10 \%$ of admissions to intensive care units. Hence, sepsis remains a major challenge in clinical practice with considerable morbidity and mortality despite modern treatments ${ }^{[2]}$. 
Early diagnosis of sepsis prior to the onset of clinical deterioration is of particular interest because an early diagnosis increases chances for an early and specific treatment. Indeed, the time taken to initiate therapy seems to be crucial and represents the major determining factor for surviving sepsis. In clinical practice, sepsis can be difficult to distinguish from other non-infectious, systemic, inflammatory responses. Blood culture is frequently used as the "gold standard" diagnostic method for sepsis. However, it usually takes 3 to 7 days to obtain the results and frequently yields low positive results. Various biomarkers have been studied for diagnosing sepsis ${ }^{[3]}$. The state of the art in diagnosing and monitoring sepsis, severe sepsis and septic shock consists of the measurement of plasma C-reactive protein (CRP) and procalcitonin (PCT) at the onset and in the course of the disease ${ }^{[4]}$. CRP and PCT in combination are clinically significant in diagnosing and monitoring sepsis ${ }^{[5]}$. Regardless of its usefulness in diagnosing sepsis, blood CRP levels were found to be elevated in many noninfectious conditions, such as autoimmune and rheumatic disorders, acute coronary syndromes, and malignant tumors post-surgery. Likewise, as with CRP, there are a number of noninfectious causes that may result in increased blood PCT levels apart from bacterial infections, such as major surgery, severe trauma, or burns ${ }^{[6]}$. Thus, there is a pressing need for new sepsis biomarkers with higher accuracy ${ }^{[7]}$.

The cluster of differentiation 14 (CD14) is a specific high-affinity receptor for lipopolysaccharides (LPSs) and LPS-binding proteins (LBPs). It is involved in the recognition of a wide variety of bacterial products. Upon binding of the LBP complex, CD14 activates the tolllike receptor 4 (TLR4)-specific proinflammatory signaling cascade, thereby starting the inflammatory reaction of the host against infectious agents. The complex LPS-LBP-CD14 is released into circulation by shedding of CD14 from the cell membrane yielding soluble CD14 (sCD14). The circulating plasma proteases activates a cleavage of $\mathrm{sCD} 14$, generating a truncated form of 64 amino acid residues named sCD14 subtype (sCD14-ST), recently renamed presepsin. The biological role of presepsin has not been exhaustively elucidated; it is a regulatory factor capable of modulating cellular and humoral immune responses by interacting directly with $\mathrm{T}$ and $\mathrm{B}$ cells. One of the production mechanisms of presepsin is related to the phagocytosis process in response to bacterial infection and cleavage of membrane CD14 with lysozomal enzymes of granulocytes ${ }^{[8]}$.

Although there is a growing body of evidence suggesting that the concentration of blood presepsin was found to be increased in patients with sepsis compared to healthy individuals and patients with SIRS ${ }^{[9]}$, the clinical significance of presepsin in the diagnosis of sepsis, evaluating sepsis severity and monitoring clinical responses to therapeutic interventions remains incompletely established. In view of the clinical importance of the early diagnosis of sepsis prior to the onset of clinical deterioration, the present study was designed to compare the diagnostic and prognostic value of presepsin, PCT and CRP in patients with SIRS. Furthermore, the usefulness of these biomarkers in monitoring clinical response to therapeutic interventions was examined.

\section{Subjects and Methods \\ Study design}

Fifty-one patients who met at least two criteria for SIRS and in whom sepsis was suspected to be the primary or concurrent diagnosis, who were admitted to the intensive care unit (ICU) at El- Maadi Military Hospital, Cairo, Egypt, were enrolled in this study. In addition, 25 healthy subjects, who underwent routine medical checkups at El-Maadi Military Hospital and had no medical history of SIRS, were enrolled as controls. A written informed consent was obtained from all the enrolled patients and healthy subjects prior to inclusion into the study in accordance with the Declaration of Helsinki adopted in 1964 and revised in 2000. The study protocol was approved by the scientific ethical committee of Faculty of Science, Ain Shams University, Cairo, Egypt.

\section{Clinical diagnosis and outcome assessment}

The diagnosis of SIRS and sepsis was made according to the seven criteria set forth by the American college of chest physicians/society of critical care medicine (ACCP/SCCM) ${ }^{[1]}$. The acute physiology and chronic health evaluation II score (APACHE II score) was used as an index of sepsis severity ${ }^{[10]}$. Demographics (age and sex) as well as past medical history of the patients were recorded on admission. Blood, urine, sputum and other cultures were performed to identify the pathogenic microorganism. For outcome assessment, a follow-up examination was planned at the $28^{\text {th }}$ day after inclusion into the study. Patients who survived until follow-up were counted as survivors, whereas patients who died within the follow-up period were counted as nonsurvivors.

\section{Measurements of plasma presepsin, PCT and CRP levels}

Venous blood samples were collected into ethylenediaminetetraacetic acid (EDTA) - vacutainer tubes (BD diagnostics, Franklin Lakes, NJ, USA) a total of five times, i.e., on admission, and 1, 3, 7 and 15 days later. Plasma samples were immediately obtained, aliquoted in sterile polypropylene tubes and stored at $-80^{\circ} \mathrm{C}$ until analysis. Plasma presepsin levels were serially measured using a compact automated immunoanalyzer, PATHFAST (Mitsubishi Chemical Europe GmbH, Düsseldorf, Germany), based on a chemiluminescent enzyme immunoassay according to the manufacturer's instructions [11]. Briefly, the PATHFAST Presepsin kit includes cartridges composed of 16 wells, containing all needed reagents, that is, magnetic particles coated with mouse monoclonal antibodies against presepsin, alkaline phosphatase (ALP)-labeled rabbit polyclonal antibodies, chemiluminescent substrate, incubation buffers and washing 
solutions. Presepsin in the sample binds to the antipresepsin antibodies to form an immunocomplex with the ALP-labeled antibodies and the antibody-coated magnetic particles. After removal of the unbound ALPlabeled antibodies using MAGTRATION Technology (magnetic particles are directly washed in the dispensing cap; Precision System Science Europe $\mathrm{GmbH}$, Wörrstadt, Germany), a chemiluminescent substrate is added to the immunocomplex. After a 10-minute incubation period, the luminescence generated by the enzyme reaction is detected by a photomultiplier to calculate the concentration of presepsin in the samples using the measurement of light emission. Ichroma PCT Test and Ichroma CRP Test (Boditech Med Inc., Kangwon, South Korea) were used for the serial measurement of plasma PCT and CRP concentrations, respectively, based on a sandwich fluorescence immunoassay following the manufacturer's instructions. In brief, the sample was mixed with the detector buffer in the test vial, and the fluorescence-labeled detector anti-PCT/anti-CRP antibody in the buffer binds to PCT/CRP antigen in the sample. The sample mixture was then loaded and migrates on the matrix of test cartridge. The complexes of detector antibody and PCT/CRP are captured to anti-PCT/anti-CRP sandwich pair antibody that has been immobilized on test matrix to measure the PCT/CRP levels.

\section{Statistical analysis}

Categorical variables were reported as the number of cases (percentage), whereas continuous variables were expressed as mean \pm standard deviation (SD) if normally distributed or as median (interquartile range, IQR: $25^{\text {th }}$ quartile to $75^{\text {th }}$ quartile or minimum-maximum as appropriate) if non-normally distributed. A 2- sided probability $(P)$ value of $<0.05$ was considered statistically significant. Changes in biomarker concentrations over time were analyzed. The correlation between biomarker levels was analyzed using Spearman's rank correlation coefficient. Receiver operating characteristic (ROC) curve analysis was performed to determine the diagnostic and prognostic value of presepsin, PCT and CRP levels. Data statistical analyses were performed using the statistical package for the social sciences (SPSS Statistics for Windows, Version 20.0; IBM Corp., Armonk, NY, USA).

\section{Results}

\section{Demographic and clinical characteristics of the study} population on admission

The study population consisted of a total of 76 subjects, including 51 SIRS patients as well as 25 healthy subjects. SIRS patients who revealed a microbiologically proven infection (positive blood cultures) were assigned to the sepsis group ( $\mathrm{n}=33$ ), whereas SIRS patients with negative blood cultures were assigned to the non-sepsis group $(n=18)$. The 25 healthy controls composed of $15(60 \%)$ male and $10(40 \%)$ female; with a mean age of $48.7 \pm 14.4$ years (range 2374). The demographic and clinical characteristics as well as microbiological findings (blood, urine, sputum and other cultures) and backgrounds of the SIRS patients stratified by the presence or absence of sepsis are summarized in Table 1. There were no statistically significant differences regarding sex $(P=0.311)$ and age $(P=0.768)$ between healthy controls and SIRS patients groups.

Levels of presepsin, PCT and CRP in the study population

On admission, presepsin, PCT and CRP levels were significantly higher in SIRS patients than in healthy controls (median [IQR]: presepsin, 921 [391-1282] vs. 258 [168-342] pg/ml; PCT, 0.80 [0.08-4.20] vs. 0.07 [0.05-0.09] ng/ml; CRP, 73.0 [34.0-142.0] vs. 3.4 $[2.8-4.8] \mathrm{mg} / \mathrm{ml}, P<0.001)$.

Kinetics of presepsin, PCT and CRP levels during ICU stay

The role of presepsin, PCT and CRP in monitoring patients with SIRS was evaluated by the analysis of serial biomarker measurements, which were performed on admission and at days 1, 3, 7 and 15. As shown in Table 2, compared with the non-sepsis group, patients with sepsis revealed significantly higher presepsin and PCT concentrations on admission and at all the subsequent time points $(P<0.05)$. On the other hand, no significant difference regarding CRP levels on admission was noted between the sepsis and non-sepsis groups $(P=0.168)$. CRP levels in patients with sepsis were significantly higher than those in patients without sepsis at days 1 and 15 . In addition, there was a trend towards higher CRP levels in patients with sepsis at days 3 and 7 $(P=0.052, P=0.057$, respectively). Presepsin displayed the highest concentration on day 1 and day 3 in the nonsepsis group and sepsis group, respectively. On the other hand, PCT and CRP showed the highest concentration on day 7 in both groups. Serial measurements analysis revealed that in the early stage of SIRS, presepsin reached the peak fast compared to PCT and CRP, not only in the septic patients but also in the non-septic patients. In addition, presepsin concentration tended to decrease on day 3 and day 7 in the non-sepsis group and sepsis group, respectively, whereas PCT and CRP concentrations tended to decrease on day 15 in both groups. In non-surviving septic patients, presepsin and PCT levels were significantly increased over 15 days. The same tendency was observed for CRP levels, but the increase did not reach statistical significance. On the other hand, in surviving septic patients, presepsin and PCT levels remained high over 7 days and tended to decrease on day 15, whereas CRP levels remained high over 15 days.

Correlation between presepsin, PCT and CRP levels

Presepsin level was positively correlated with PCT and CRP levels not only in the septic patients but also in the non-septic patients on admission and at days 1, 3, 7 and 15 (Table 3).

Diagnostic accuracy of presepsin, PCT and CRP

The diagnostic performance of presepsin, PCT and CRP was assessed by ROC curve analysis in the septic versus non-septic groups (Table 4, Figure1). 
Table 1: Demographic and clinical characteristics of SIRS patients.

\begin{tabular}{|c|c|c|c|c|}
\hline \multirow[b]{2}{*}{ Characteristics } & \multirow[b]{2}{*}{$\begin{array}{l}\text { SIRS patients } \\
\quad(n=51)\end{array}$} & \multicolumn{2}{|c|}{$\begin{array}{l}\text { SIRS patients }(n=51) \\
\end{array}$} & \multirow{2}{*}{$\begin{array}{c}P \\
\text { value }\end{array}$} \\
\hline & & $\begin{array}{l}\text { Patients with sepsis } \\
\quad(n=33)\end{array}$ & $\begin{array}{c}\text { Patients without sepsis } \\
(\mathrm{n}=18)\end{array}$ & \\
\hline $\begin{array}{l}\text { Gender } \\
\text { Male, n }(\%) \\
\text { Female, n }(\%) \\
\end{array}$ & $\begin{array}{l}35(68.6) \\
16(31.4) \\
\end{array}$ & $\begin{array}{l}23(69.7) \\
10(30.3) \\
\end{array}$ & $\begin{array}{c}12(66.7) \\
6(33.3) \\
\end{array}$ & 0.532 \\
\hline Age (years) & $\begin{array}{c}49.8 \pm 16.1 \\
(20-77) \\
\end{array}$ & $\begin{array}{c}55.2 \pm 14.6 \\
(21-77) \\
\end{array}$ & $\begin{array}{c}40 \pm 14.2 \\
(20-64) \\
\end{array}$ & $0.001 *$ \\
\hline $\begin{array}{l}\text { Cigarette smoking status } \\
\text { Smokers, } \mathrm{n}(\%) \\
\text { Non-smokers, } \mathrm{n}(\%)\end{array}$ & $\begin{array}{l}27(52.9) \\
24(47.1)\end{array}$ & $\begin{array}{l}17(51.5) \\
16(48.5)\end{array}$ & $\begin{array}{c}10(55.6) \\
8(44.4)\end{array}$ & 0.508 \\
\hline \multicolumn{5}{|l|}{ Patient backgrounds } \\
\hline $\begin{array}{l}\text { Diabetes mellitus } \\
\text { Yes, } \mathrm{n}(\%) \\
\text { No, } \mathrm{n}(\%)\end{array}$ & $\begin{array}{l}31(60.8) \\
20(39.2)\end{array}$ & $\begin{array}{c}24(72.7) \\
9(27.3) \\
\end{array}$ & $\begin{array}{c}7(38.9) \\
11(61.1) \\
\end{array}$ & $0.020^{*}$ \\
\hline $\begin{array}{l}\text { Hypertension } \\
\text { Yes, } \mathrm{n}(\%) \\
\text { No, } \mathrm{n}(\%) \\
\end{array}$ & $\begin{array}{l}30(58.8) \\
21(41.2) \\
\end{array}$ & $\begin{array}{l}25(75.8) \\
8(24.2) \\
\end{array}$ & $\begin{array}{c}5(27.8) \\
13(72.2) \\
\end{array}$ & $0.001^{*}$ \\
\hline $\begin{array}{l}\text { CVD } \\
\text { Yes, n }(\%) \\
\text { No, n }(\%) \\
\end{array}$ & $\begin{array}{r}8(15.7) \\
43(84.3) \\
\end{array}$ & $\begin{array}{c}7(21.2) \\
26(78.8) \\
\end{array}$ & $\begin{array}{c}1(5.6) \\
17(94.4) \\
\end{array}$ & 0.143 \\
\hline $\begin{array}{l}\text { Liver diseases } \\
\text { Yes, } \mathrm{n}(\%) \\
\text { No, } \mathrm{n}(\%) \\
\end{array}$ & $\begin{array}{r}9(17.6) \\
42(82.4) \\
\end{array}$ & $\begin{array}{r}7(21.2) \\
26(78.8) \\
\end{array}$ & $\begin{array}{c}2(11.1) \\
16(88.9) \\
\end{array}$ & 0.310 \\
\hline $\begin{array}{l}\text { Kidney diseases } \\
\text { Yes, } \mathrm{n}(\%) \\
\text { No, } \mathrm{n}(\%)\end{array}$ & $\begin{array}{l}11(21.6) \\
40(78.4)\end{array}$ & $\begin{array}{l}10(30.3) \\
23(69.7)\end{array}$ & $\begin{array}{c}1(5.6) \\
17(94.4)\end{array}$ & $0.039 *$ \\
\hline $\begin{array}{l}\text { COPD } \\
\text { Yes, n }(\%) \\
\text { No, } \mathrm{n}(\%) \\
\end{array}$ & $\begin{array}{r}8(15.7) \\
43(84.3) \\
\end{array}$ & $\begin{array}{c}5(15.2) \\
28(84.8) \\
\end{array}$ & $\begin{array}{c}3(16.7) \\
15(83.3) \\
\end{array}$ & 0.591 \\
\hline $\begin{array}{l}\text { Previous ICU admission } \\
\text { Yes, } \mathrm{n}(\%) \\
\text { No, } \mathrm{n}(\%)\end{array}$ & $\begin{array}{l}27(52.9) \\
24(47.1) \\
\end{array}$ & $\begin{array}{l}21(63.6) \\
12(36.4) \\
\end{array}$ & $\begin{array}{c}6(33.3) \\
12(66.7) \\
\end{array}$ & $0.037 *$ \\
\hline \multicolumn{5}{|l|}{ Microbiology findings } \\
\hline \multicolumn{5}{|l|}{ Blood culture } \\
\hline $\begin{array}{l}\text { Day 1 } \\
\text { Positive, n (\%) } \\
\text { Negative, n (\%) } \\
\end{array}$ & $\begin{aligned} 2(3.9) \\
49(96.1) \\
\end{aligned}$ & $\begin{array}{c}2(6.1) \\
31(93.9) \\
\end{array}$ & $\begin{array}{c}0(0.0) \\
18(100.0) \\
\end{array}$ & 0.414 \\
\hline $\begin{array}{l}\text { Day } 7 \\
\text { Positive, n (\%) } \\
\text { Negative, n (\%) } \\
\end{array}$ & $\begin{array}{l}10(19.6) \\
41(80.4) \\
\end{array}$ & $\begin{array}{l}10(30.3) \\
23(69.7)\end{array}$ & $\begin{array}{c}0(0.0) \\
18(100.0) \\
\end{array}$ & $0.007 *$ \\
\hline \multicolumn{5}{|l|}{ Urine culture } \\
\hline $\begin{array}{l}\text { Day 1 } \\
\text { Positive, n (\%) } \\
\text { Negative, n (\%) } \\
\end{array}$ & $\begin{array}{c}9(17.6) \\
42(82.4) \\
\end{array}$ & $\begin{array}{c}8(24.2) \\
25(75.8) \\
\end{array}$ & $\begin{array}{c}1(5.6) \\
17(94.4) \\
\end{array}$ & 0.095 \\
\hline $\begin{array}{l}\text { Day } 7 \\
\text { Positive, n (\%) } \\
\text { Negative, n (\%) }\end{array}$ & $\begin{array}{l}14(27.5) \\
37(72.5)\end{array}$ & $\begin{array}{l}13(39.4) \\
20(60.6)\end{array}$ & $\begin{array}{c}1(5.6) \\
17(94.4)\end{array}$ & $0.009^{*}$ \\
\hline \multicolumn{5}{|l|}{ Sputum culture } \\
\hline $\begin{array}{l}\text { Day } 1 \\
\text { Positive, n (\%) } \\
\text { Negative, n (\%) }\end{array}$ & $\begin{array}{c}3(5.9) \\
48(94.1)\end{array}$ & $\begin{array}{c}3(9.1) \\
30(90.9)\end{array}$ & $\begin{array}{c}0(0.0) \\
18(100.0)\end{array}$ & 0.262 \\
\hline $\begin{array}{l}\text { Day } 7 \\
\text { Positive, n (\%) } \\
\text { Negative, n (\%) } \\
\end{array}$ & $\begin{array}{l}20(39.2) \\
31(60.8) \\
\end{array}$ & $\begin{array}{l}19(57.6) \\
14(42.4) \\
\end{array}$ & $\begin{array}{c}1(5.6) \\
17(94.4) \\
\end{array}$ & $<0.001 *$ \\
\hline \multicolumn{5}{|l|}{ Other cultures } \\
\hline $\begin{array}{l}\text { Day } 1 \\
\text { Positive, n (\%) } \\
\text { Negative, n (\%) } \\
\end{array}$ & $\begin{array}{c}3(5.9) \\
48(94.1) \\
\end{array}$ & $\begin{array}{c}3(9.1) \\
30(90.9) \\
\end{array}$ & $\begin{array}{c}0(0.0) \\
18(100.0)\end{array}$ & 0.262 \\
\hline $\begin{array}{l}\text { Day } 7 \\
\text { Positive, n (\%) } \\
\text { Negative, n (\%) }\end{array}$ & $\begin{array}{c}6(11.8) \\
45(88.2) \\
\end{array}$ & $\begin{array}{c}6(18.1) \\
27(81.9)\end{array}$ & $\begin{array}{c}0(0.0) \\
18(100.0)\end{array}$ & 0.061 \\
\hline $\begin{array}{l}\text { Pathogenic microorganism } \\
\text { None } \\
\text { Gram negative } \\
\text { Gram positive } \\
\text { Gram positive+ Gram negative }\end{array}$ & $\begin{array}{c}18(35.3) \\
26(51.0) \\
0(0.0) \\
7(13.7) \\
\end{array}$ & $\begin{array}{c}0(0.0) \\
26(78.8) \\
0(0.0) \\
7(21.2)\end{array}$ & $\begin{array}{c}18(100.0) \\
0(0.0) \\
0(0.0) \\
0(0.0)\end{array}$ & $<0.001 *$ \\
\hline APACHEII score on admission & $\begin{array}{c}14.7 \pm 4.4 \\
(8-28) \\
\end{array}$ & $\begin{array}{c}16.1 \pm 4.6 \\
(10-28) \\
\end{array}$ & $\begin{array}{c}12.1 \pm 2.6 \\
(8-18) \\
\end{array}$ & $0.002 *$ \\
\hline $\begin{array}{l}\text { 28-day mortality rate, n (\%) } \\
\text { Non-surviving patients, n (\%) } \\
\text { Surviving patients, n (\%) }\end{array}$ & $\begin{array}{l}20(39.2) \\
31(60.8)\end{array}$ & $\begin{array}{l}19(57.6) \\
14(42.4)\end{array}$ & $\begin{array}{c}1(5.6) \\
17(94.4)\end{array}$ & $<0.001 *$ \\
\hline
\end{tabular}

SIRS, systemic inflammatory response syndrome; CVD, cardiovascular disease; COPD; chronic obstructive pulmonary disease, APACHEII; acute physiology and chronic health evaluation II score. Qualitative data are represented as the number of cases (\%), whereas quantitative data are represented as mean $\pm \mathrm{SD}$ (range, minimum-maximum). * indicates a statistically significant difference. 
Table 2: Serial measurements of presepsin, PCT and CRP levels on admission and at days 1, 3, 7 and 15 in SIRS patients stratified by the presence or absence of sepsis.

\begin{tabular}{|c|c|c|c|c|c|c|c|c|}
\hline \multirow{2}{*}{\multicolumn{2}{|c|}{ Biomarkers }} & \multirow{3}{*}{$\begin{array}{c}\begin{array}{c}\text { SIRS patients } \\
(\mathbf{n}=\mathbf{5 1})\end{array} \\
921 \\
(391-1282)\end{array}$} & \multicolumn{2}{|c|}{$\begin{array}{c}\text { SIRS patients } \\
(\mathrm{n}=51)\end{array}$} & \multirow{3}{*}{$\begin{array}{l}\boldsymbol{P} \text { value } \\
<0.001 *\end{array}$} & \multicolumn{2}{|c|}{$\begin{array}{c}\text { Patients with sepsis } \\
(\mathrm{n}=33)\end{array}$} & \multirow{3}{*}{$\begin{array}{l}\boldsymbol{P} \text { value } \\
<0.001 *\end{array}$} \\
\hline & & & \multirow{2}{*}{$\begin{array}{c}\begin{array}{c}\text { Patients without } \\
\text { sepsis (n=18) }\end{array} \\
472.0 \\
(216.0-878.75)\end{array}$} & \multirow{2}{*}{$\begin{array}{c}\begin{array}{c}\text { Patients with } \\
\text { sepsis }(\mathbf{n}=33)\end{array} \\
1183.0 \\
(615.5-1475.0) \\
\end{array}$} & & \multirow{2}{*}{$\begin{array}{c}\begin{array}{c}\text { Non-surviving } \\
\text { patients }(\mathbf{n}=19)\end{array} \\
1301.0 \\
(1183.0-1908.0) \\
\end{array}$} & \multirow{2}{*}{$\begin{array}{c}\begin{array}{c}\text { Surviving } \\
\text { patients }(\mathbf{n}=14)\end{array} \\
615.5 \\
(388.3-923.3) \\
\end{array}$} & \\
\hline .气 & $\begin{array}{c}\text { Pesepsin } \\
(\mathrm{pg} / \mathrm{ml})\end{array}$ & & & & & & & \\
\hline 尊 & $\begin{array}{c}\text { PCT } \\
(\mathbf{n g} / \mathbf{m l})\end{array}$ & $\begin{array}{c}0.80 \\
(0.08-4.20) \\
\end{array}$ & $\begin{array}{c}0.09 \\
(0.05-0.455) \\
\end{array}$ & $\begin{array}{c}1.30 \\
(0.095-7) \\
\end{array}$ & $0.001^{*}$ & $\begin{array}{c}6.10 \\
(3.70-8.98) \\
\end{array}$ & $\begin{array}{c}0.01 \\
(0.07-0.83) \\
\end{array}$ & $<0.001 *$ \\
\hline$\tilde{\sigma}$ & $\begin{array}{c}\text { CRP } \\
(\mathrm{mg} / \mathrm{ml})\end{array}$ & $\begin{array}{c}73.0 \\
(34.0-142.0) \\
\end{array}$ & $\begin{array}{c}72.8 \\
(24.0-93.0) \\
\end{array}$ & $\begin{array}{c}78.0 \\
(39.5-199.0) \\
\end{array}$ & 0.168 & $\begin{array}{c}71.0 \\
(34.0-183.0) \\
\end{array}$ & $\begin{array}{c}78.0 \\
(54.8-216.0) \\
\end{array}$ & 0.602 \\
\hline \multirow{3}{*}{$\overrightarrow{\mathrm{a}}$} & $\begin{array}{c}\text { Pesepsin } \\
(\mathrm{pg} / \mathrm{ml})\end{array}$ & $\begin{array}{c}981 \\
(542-1435)\end{array}$ & $\begin{array}{c}721.0 \\
(293.25-978.75)\end{array}$ & $\begin{array}{c}1192.0 \\
(641.5-1831.0)\end{array}$ & $0.002^{*}$ & $\begin{array}{c}1448.0 \\
(1203-2409)\end{array}$ & $\begin{array}{c}733.0 \\
(509.5-1063.0)\end{array}$ & $<0.001 *$ \\
\hline & $\begin{array}{c}\text { PCT } \\
\text { (ng/ml) }\end{array}$ & $\begin{array}{c}0.9 \\
(0.09-5.78)\end{array}$ & $\begin{array}{c}0.09 \\
(0.065-0.81)\end{array}$ & $\begin{array}{c}1.80 \\
(0.16-8.705)\end{array}$ & $0.001^{*}$ & $\begin{array}{c}8.20 \\
(3.90-11.50)\end{array}$ & $\begin{array}{c}0.30 \\
(0.08-1.43)\end{array}$ & $<0.001 *$ \\
\hline & $\underset{(\mathrm{mg} / \mathrm{ml})}{\mathrm{CRP}}$ & $\begin{array}{c}92.0 \\
(57-178)\end{array}$ & $\begin{array}{c}80.5 \\
(48.0-102.3)\end{array}$ & $\begin{array}{c}112.0 \\
(58.0-241.0)\end{array}$ & $0.026^{*}$ & $\begin{array}{c}123.0 \\
(57.0-243.0)\end{array}$ & $\begin{array}{c}110.5 \\
(76.5-239.75)\end{array}$ & 0.982 \\
\hline \multirow{3}{*}{$\sum^{\infty}$} & $\begin{array}{c}\text { Pesepsin } \\
(\mathrm{pg} / \mathrm{ml})\end{array}$ & $\begin{array}{c}1134.0 \\
(412.0-2045.0)\end{array}$ & $\begin{array}{c}464 \\
(304-1178.5)\end{array}$ & $\begin{array}{c}1972 \\
(771-2796.5)\end{array}$ & $<0.001 *$ & $\begin{array}{c}2514.0 \\
(1782.0-2914.0)\end{array}$ & $\begin{array}{c}771.0 \\
(397.8-2013.0)\end{array}$ & $<0.001 *$ \\
\hline & $\begin{array}{c}\text { PCT } \\
(\mathbf{n g} / \mathbf{m l})\end{array}$ & $\begin{array}{c}1.3 \\
(0.09-8.1)\end{array}$ & $\begin{array}{c}0.11 \\
(0.07-1.45) \\
\end{array}$ & $\begin{array}{c}5.1 \\
(0.47-12.47) \\
\end{array}$ & $<0.001 *$ & $\begin{array}{c}10.9 \\
(6.3-17.1) \\
\end{array}$ & $\begin{array}{c}0.655 \\
0.12-2.9425) \\
\end{array}$ & $<0.001 *$ \\
\hline & $\begin{array}{c}\text { CRP } \\
(\mathrm{mg} / \mathrm{ml})\end{array}$ & $\begin{array}{c}159.0 \\
(91.0-2190) \\
\end{array}$ & $\begin{array}{c}115.5 \\
(52.5-187.3) \\
\end{array}$ & $\begin{array}{c}181.0 \\
(94.0-268.0) \\
\end{array}$ & 0.052 & $\begin{array}{c}189.0 \\
(92.0-297.0) \\
\end{array}$ & $\begin{array}{c}162.0 \\
(93.8-198.3) \\
\end{array}$ & 0.199 \\
\hline \multirow{3}{*}{$\hat{\theta}$} & $\begin{array}{c}\text { Pesepsin } \\
\text { (pg/ml) }\end{array}$ & $\begin{array}{c}941.0 \\
(407.5-2417.0) \\
\end{array}$ & $\begin{array}{c}417.0 \\
(310.0-715.5) \\
\end{array}$ & $\begin{array}{c}1928.5 \\
(799.8-3241.0) \\
\end{array}$ & $<0.001 *$ & $\begin{array}{c}3191.0 \\
(2105.25-3960)\end{array}$ & $\begin{array}{c}816.5 \\
(507-1521.25) \\
\end{array}$ & $<0.001 *$ \\
\hline & $\begin{array}{c}\text { PCT } \\
(\mathbf{n g} / \mathbf{m l})\end{array}$ & $\begin{array}{c}1.9 \\
(0.1-9.7) \\
\end{array}$ & $\begin{array}{c}0.21 \\
(0.05-1.38) \\
\end{array}$ & $\begin{array}{c}6.7 \\
(0.8475-13.65) \\
\end{array}$ & $<0.001^{*}$ & $\begin{array}{c}12.8 \\
(9.1-19.3) \\
\end{array}$ & $\begin{array}{c}0.87 \\
(0.085-4.10) \\
\end{array}$ & $<0.001 *$ \\
\hline & $\underset{(\mathrm{mg} / \mathrm{ml})}{\mathbf{C R P}}$ & $\begin{array}{c}189 \\
(112-229.25)\end{array}$ & $\begin{array}{c}145.5 \\
(69.5-200.0)\end{array}$ & $\begin{array}{c}213.0 \\
(123.3-281.0)\end{array}$ & 0.057 & $\begin{array}{c}213.0 \\
(152.8-321.0)\end{array}$ & $\begin{array}{c}166.0 \\
(108.5-281.0)\end{array}$ & 0.319 \\
\hline \multirow{3}{*}{$\frac{10}{2}$} & $\begin{array}{c}\text { Pesepsin } \\
(\mathrm{pg} / \mathrm{ml})\end{array}$ & $\begin{array}{c}941.0 \\
(407.5-2417.0)\end{array}$ & $\begin{array}{c}217.0 \\
(189-646.5)\end{array}$ & $\begin{array}{c}759.0 \\
(420.3-1719.3)\end{array}$ & $0.001^{*}$ & $\begin{array}{c}2934.5 \\
(1580.5-4147.8)\end{array}$ & $\begin{array}{c}560.5 \\
(296.5-837.3)\end{array}$ & $<0.001 *$ \\
\hline & $\begin{array}{c}\text { PCT } \\
(\mathbf{n g} / \mathbf{m l})\end{array}$ & $\begin{array}{c}1.9 \\
(0.1-9.7)\end{array}$ & $\begin{array}{c}0.08 \\
(0.05-0.68)\end{array}$ & $\begin{array}{c}2.255 \\
(0.17-9.9) \\
\end{array}$ & $0.004^{*}$ & $\begin{array}{c}16.5 \\
(7.4-18.3) \\
\end{array}$ & $\begin{array}{c}0.45 \\
(0.05-2.54) \\
\end{array}$ & $<0.001 *$ \\
\hline & $\underset{(\mathrm{mg} / \mathrm{ml})}{\mathrm{CRP}}$ & $\begin{array}{c}173 \\
(96-258)\end{array}$ & $\begin{array}{c}132.0 \\
(82.5-193.5)\end{array}$ & $\begin{array}{c}210 \\
(128.6-279.7)\end{array}$ & $0.015^{*}$ & $\begin{array}{c}244.0 \\
(157.0-394.0)\end{array}$ & $\begin{array}{c}201.5 \\
(117.6-275.5)\end{array}$ & 0.494 \\
\hline
\end{tabular}

SIRS, systemic inflammatory response syndrome; PCT, procalcitonin, CRP, C-reactive protein. Data are represented as median (interquartile range, IQR: $25^{\text {th }}$ quartile to $75^{\text {th }}$ quartile). * indicates a statistically significant difference.

The area under the ROC curves (AUCs) for presepsin, PCT, and CRP were $0.805,0.780$ and 0.613 , respectively, indicating that the value of presepsin to diagnose sepsis was comparable to that of PCT (AUC, 0.805 vs. $0.780 ; P=0.755$ ), whereas presepsin performed significantly better than CRP for diagnosing sepsis (AUC, 0.805 vs. $0.613 ; P=0.029$ ). On the other hand, there was a trend towards a better diagnostic performance of PCT than CRP in differentiating patients with septic SIRS from patients with non-septic SIRS (AUC, 0.780 vs. $0.613 ; P=0.062$ ).

Prognostic value of presepsin, PCT, CRP and APACHE II score

In patients with sepsis, presepsin and PCT levels on admission were significantly higher in non-survivors $(\mathrm{n}=19)$ than in survivors $(\mathrm{n}=14) \quad(P<0.001)$, whereas CRP levels on admission did not differ significantly between the surviving and non-surviving groups $(P=0.602)$ (Table 2). Furthermore, the APACHE II score on admission were significantly higher in nonsurvivors than in survivors (mean $\pm \mathrm{SD}, 17.9 \pm 4.9$
[11-28] vs. $13.6 \pm 3[10-19], P=0.007)$. All septic patients were followed-up for 28 days after enrollment in the study, and the performance of presepsin, PCT, CRP and APACHE II score for predicting 28-day all-cause inhospital mortality was evaluated by ROC curve analysis in the surviving versus non-surviving groups (Table 5, Figure 2). PCT displayed the best performance for predicting 28-day mortality with the highest AUC value (AUC $=0.932$ ). Additionally, presepsin with an AUC of 0.891 did not show better prognostic performance than PCT (AUC, 0.932 vs. $0.891 ; P=0.465$ ). Furthermore, PCT performed significantly better than CRP (AUC $=0.445)$ and APACHE II score $(\mathrm{AUC}=0.765)$ $(P<0.001)$. On the other hand, the AUC of presepsin was slightly higher than that of the APACHE II score $(0.891$ vs. $0.765 ; P=0.087$ ), indicating that presepsin might perform better than APACHE II score in predicting 28day mortality. Likewise, presepsin performed significantly better than CRP in predicting 28-day mortality ( 0.891 vs. $0.445 ; P<0.001)$. 
Table 3: Correlation between presepsin, PCT and CRP levels on admission and at days 1, 3, 7 and 15 in patients with or without sepsis.

\begin{tabular}{|c|c|c|c|c|c|c|c|c|c|c|c|c|c|}
\hline & \multirow{3}{*}{ iables } & \multicolumn{4}{|c|}{ Pesepsin (pg/ml) } & \multicolumn{4}{|c|}{ PCT (ng/ml) } & \multicolumn{4}{|c|}{ CRP (mg/ml) } \\
\hline & & \multicolumn{2}{|c|}{$\begin{array}{c}\text { Patients } \\
\text { with sepsis } \\
(\mathbf{n}=33)\end{array}$} & \multicolumn{2}{|c|}{$\begin{array}{c}\text { Patients } \\
\text { without sepsis } \\
(\mathrm{n}=18)\end{array}$} & \multicolumn{2}{|c|}{$\begin{array}{c}\text { Patients } \\
\text { with sepsis } \\
(\mathbf{n}=33)\end{array}$} & \multicolumn{2}{|c|}{$\begin{array}{c}\text { Patients } \\
\text { without sepsis } \\
(n=18)\end{array}$} & \multicolumn{2}{|c|}{$\begin{array}{c}\text { Patients } \\
\text { with sepsis } \\
(\mathrm{n}=33)\end{array}$} & \multicolumn{2}{|c|}{$\begin{array}{c}\text { Patients } \\
\text { without sepsis } \\
(n=18)\end{array}$} \\
\hline & & $\mathbf{R}$ & $\begin{array}{c}P \\
\text { value }\end{array}$ & $\mathbf{R}$ & $\begin{array}{c}P \\
\text { value }\end{array}$ & $\mathbf{R}$ & $\begin{array}{c}P \\
\text { value }\end{array}$ & $\mathbf{R}$ & $\begin{array}{c}P \\
\text { value }\end{array}$ & $\mathbf{R}$ & $\begin{array}{c}P \\
\text { value }\end{array}$ & $\mathbf{R}$ & $\begin{array}{c}P \\
\text { value }\end{array}$ \\
\hline \multirow{3}{*}{ 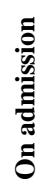 } & Pesepsin & & & & & 0.773 & $<0.001 *$ & 0.826 & $<0.001 *$ & 0.128 & 0.478 & 0.272 & 0.274 \\
\hline & PCT & 0.773 & $<0.001 *$ & 0.826 & $<0.001^{*}$ & & & & & 0.010 & 0.958 & 0.379 & 0.121 \\
\hline & CRP & 0.128 & 0.478 & 0.272 & 0.274 & 0.010 & 0.958 & 0.379 & 0.121 & & & & \\
\hline \multirow{3}{*}{$\vec{\Xi}$} & Pesepsin & & & & & 0.758 & $<0.001^{*}$ & 0.617 & $0.006^{*}$ & 0.172 & 0.339 & 0.371 & 0.130 \\
\hline & PCT & 0.758 & $<0.001 *$ & 0.617 & $0.006^{*}$ & & & & & 0.171 & 0.341 & 0.681 & $0.002 *$ \\
\hline & CRP & 0.172 & 0.339 & 0.371 & 0.130 & 0.171 & 0.341 & 0.681 & $0.002 *$ & & & & \\
\hline \multirow{3}{*}{$\stackrel{\infty}{\overparen{E}}$} & Pesepsin & & & & & 0.807 & $<0.001 *$ & 0.894 & $<0.001^{*}$ & 0.512 & $0.030 *$ & 0.670 & $<0.001 *$ \\
\hline & PCT & 0.807 & $<0.001 *$ & 0.894 & $<0.001^{*}$ & & & & & 0.661 & $0.003 *$ & 0.488 & $0.004 *$ \\
\hline & CRP & 0.670 & $<0.001 *$ & 0.512 & $0.030^{*}$ & 0.488 & $0.004 *$ & 0.661 & $0.003 *$ & & & & \\
\hline \multirow{3}{*}{ है } & Pesepsin & & & & & 0.921 & $<0.001 *$ & 0.757 & $<0.001^{*}$ & 0.516 & $0.002 *$ & 0.555 & $0.017^{*}$ \\
\hline & PCT & 0.921 & $<0.001 *$ & 0.757 & $<0.001^{*}$ & & & & & 0.445 & $0.011^{*}$ & 0.438 & 0.069 \\
\hline & CRP & 0.516 & $0.002 *$ & 0.555 & $0.017^{*}$ & 0.445 & $0.011 *$ & 0.438 & 0.069 & & & & \\
\hline \multirow{3}{*}{$\frac{1}{2}$} & Pesepsin & & & & & 0.855 & $<0.001 *$ & 0.704 & $0.002 *$ & 0.433 & 0.057 & 0.591 & $0.012 *$ \\
\hline & PCT & 0.855 & $<0.001^{*}$ & 0.704 & $0.002 *$ & & & & & 0.474 & $0.035^{*}$ & 0.712 & $0.001 *$ \\
\hline & CRP & 0.433 & 0.057 & 0.591 & $0.012 *$ & 0.474 & $0.035^{*}$ & 0.712 & $0.001 *$ & & & & \\
\hline
\end{tabular}

PCT, procalcitonin, CRP, C-reactive protein. The correlation was analyzed using Spearman's rank correlation coefficient. * indicates a statistically significant correlation.

Table 4: Cut-off values, sensitivity, specificity, positive predictive value, negative predictive value and area under the curves of presepsin, PCT and CRP for diagnosing sepsis.

\begin{tabular}{|c|c|c|c|c|c|c|c|c|c|c|}
\hline Biomarker & $\begin{array}{c}\text { Patients } \\
\text { with } \\
\text { sepsis } \\
(\mathbf{n}=33)\end{array}$ & $\begin{array}{c}\text { Patients } \\
\text { without } \\
\text { sepsis } \\
(n=18)\end{array}$ & $\begin{array}{c}\text { Cut-off } \\
\text { point }\end{array}$ & $\begin{array}{c}\text { Sensitivity } \\
(\%)\end{array}$ & $\begin{array}{c}\text { Specificity } \\
(\%)\end{array}$ & $\begin{array}{r}\text { PPV } \\
(\%)\end{array}$ & $\begin{array}{l}\text { NPV } \\
(\%)\end{array}$ & $\begin{array}{c}\text { Accuracy } \\
(\%)\end{array}$ & $\underset{(95 \% \mathrm{CI})}{\mathrm{AUC}}$ & $\begin{array}{c}P \\
\text { value }\end{array}$ \\
\hline \multirow{2}{*}{$\begin{array}{c}\text { Pesepsin } \\
(\mathrm{pg} / \mathrm{ml})\end{array}$} & 23 & 3 & $\leq 907$ & \multirow{2}{*}{69.7} & \multirow{2}{*}{83.3} & \multirow{2}{*}{88.5} & \multirow{2}{*}{60.0} & \multirow{2}{*}{74.5} & \multirow{2}{*}{$\begin{array}{c}0.805 \\
(0.681-0.929)\end{array}$} & \multirow{2}{*}{$<0.001^{*}$} \\
\hline & 10 & 15 & $>907$ & & & & & & & \\
\hline \multirow{2}{*}{$\begin{array}{c}\text { PCT } \\
(\mathbf{n g} / \mathrm{ml})\end{array}$} & 13 & 16 & $\leq 0.85$ & \multirow{2}{*}{60.6} & \multirow{2}{*}{88.9} & \multirow{2}{*}{90.9} & \multirow{2}{*}{55.2} & \multirow{2}{*}{70.6} & \multirow{2}{*}{$\begin{array}{c}0.780 \\
(0.654-0.906)\end{array}$} & \multirow{2}{*}{$0.001 *$} \\
\hline & 20 & 2 & $>0.85$ & & & & & & & \\
\hline \multirow{2}{*}{$\underset{(\mathrm{mg} / \mathrm{ml})}{\mathrm{CRP}}$} & 19 & 15 & $\leq 97$ & \multirow{2}{*}{42.4} & \multirow{2}{*}{83.3} & \multirow{2}{*}{82.4} & \multirow{2}{*}{44.1} & \multirow{2}{*}{56.9} & \multirow{2}{*}{$\begin{array}{c}0.613 \\
(0.457-0.769)\end{array}$} & \multirow{2}{*}{0.187} \\
\hline & 14 & 3 & $>97$ & & & & & & & \\
\hline \multicolumn{4}{|c|}{$P$ value } & $\begin{array}{c}0.333^{\mathrm{a}} \\
0.004^{\mathrm{b} *} \\
0.061^{\mathrm{c}}\end{array}$ & $\begin{array}{l}0.412^{\mathrm{a}} \\
1.000^{\mathrm{b}} \\
0.412^{\mathrm{c}}\end{array}$ & $\begin{array}{l}0.690^{\mathrm{a}} \\
0.381^{\mathrm{b}} \\
0.203^{\mathrm{c}}\end{array}$ & $\begin{array}{l}0.623^{\mathrm{a}} \\
0.104^{\mathrm{b}} \\
0.259^{\mathrm{c}}\end{array}$ & $\begin{array}{l}0.659^{\mathrm{a}} \\
0.056^{\mathrm{b}} \\
0.146^{\mathrm{c}}\end{array}$ & $\begin{array}{c}0.755^{\mathrm{a}} \\
0.029^{\mathrm{b}} * \\
0.062^{\mathrm{c}}\end{array}$ & \\
\hline
\end{tabular}

PCT, procalcitonin, CRP, C-reactive protein; PPV, Positive predictive value; NPV, Negative predictive value; AUC, area under the curve; $95 \%$ CI, 95\% confidence interval. ${ }^{\text {a }}$, pesepsin vs. PCT; ${ }^{\text {b }}$, pesepsin vs. CRP; ${ }^{\text {c }}$, PCT vs. CRP. * indicates a statistically significant correlation. 


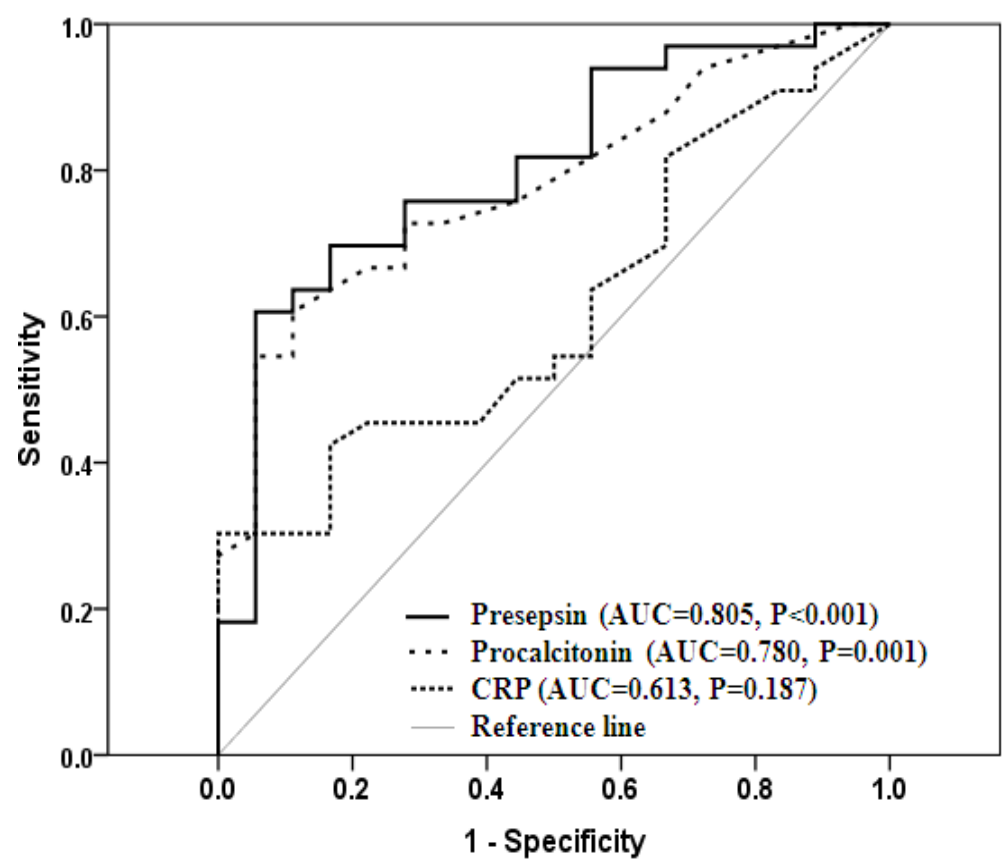

Fig. 1: Receiver operating characteristic (ROC) curves of presepsin, PCT and CRP for diagnosing sepsis.

Table 5: Cut-off values, sensitivity, specificity, positive predictive value, negative predictive value and area under the curves of presepsin, PCT and CRP for predicting 28-day mortality in patients with sepsis.

\begin{tabular}{|c|c|c|c|c|c|c|c|c|c|c|}
\hline Biomarker & $\begin{array}{l}\text { Surviving } \\
\text { patients } \\
\text { with sepsis } \\
(\mathrm{n}=14)\end{array}$ & $\begin{array}{c}\text { Non- } \\
\text { surviving } \\
\text { patients } \\
\text { with sepsis } \\
(\mathrm{n}=19) \\
\end{array}$ & $\begin{array}{l}\text { Cut-off } \\
\text { point }\end{array}$ & $\begin{array}{c}\text { Sensitivity } \\
(\%)\end{array}$ & $\begin{array}{c}\text { Specificity } \\
(\%)\end{array}$ & $\begin{array}{l}\text { PPV } \\
(\%)\end{array}$ & $\begin{array}{l}\text { NPV } \\
(\%)\end{array}$ & $\begin{array}{c}\text { Accuracy } \\
(\%)\end{array}$ & $\underset{(95 \% \text { CI })}{\text { AUC }}$ & $\begin{array}{c}P \\
\text { value }\end{array}$ \\
\hline \multirow{2}{*}{$\begin{array}{c}\text { Pesepsin } \\
(\mathrm{pg} / \mathrm{ml})\end{array}$} & 2 & 18 & $\geq 957.5$ & \multirow{2}{*}{94.7} & \multirow{2}{*}{85.7} & \multirow{2}{*}{90.0} & \multirow{2}{*}{92.3} & \multirow{2}{*}{90.9} & \multirow{2}{*}{$\begin{array}{c}0.891 \\
(0.765- \\
1.000)\end{array}$} & \multirow{2}{*}{$<0.001^{\prime}$} \\
\hline & 12 & 1 & $<957.5$ & & & & & & & \\
\hline \multirow{2}{*}{$\begin{array}{c}\text { PCT } \\
(\mathbf{n g} / \mathrm{ml})\end{array}$} & 0 & 15 & $\geq 2.60$ & \multirow{2}{*}{78.9} & \multirow{2}{*}{100.0} & \multirow{2}{*}{100.0} & \multirow{2}{*}{77.8} & \multirow{2}{*}{87.9} & \multirow{2}{*}{$\begin{array}{c}0.932 \\
(0.846- \\
1.000)\end{array}$} & \multirow{2}{*}{$<0.001^{\prime}$} \\
\hline & 14 & 4 & $<2.60$ & & & & & & & \\
\hline \multirow{2}{*}{$\underset{(\mathrm{mg} / \mathrm{ml})}{\mathbf{C R P}}$} & 5 & 9 & $\geq 97.0$ & \multirow{2}{*}{47.4} & \multirow{2}{*}{64.3} & \multirow{2}{*}{64.3} & \multirow{2}{*}{47.4} & \multirow{2}{*}{54.5} & \multirow{2}{*}{$\begin{array}{c}0.445 \\
(0.245- \\
0.646)\end{array}$} & \multirow{2}{*}{0.597} \\
\hline & 9 & 10 & $<97.0$ & & & & & & & \\
\hline \multirow{2}{*}{$\begin{array}{l}\text { APACHE } \\
\text { II score }\end{array}$} & 2 & 11 & $\geq 16.5$ & \multirow{2}{*}{57.9} & \multirow{2}{*}{85.7} & \multirow{2}{*}{84.6} & \multirow{2}{*}{60.0} & \multirow{2}{*}{69.7} & \multirow{2}{*}{$\begin{array}{l}0.765 \\
(0.598- \\
0.932)\end{array}$} & \multirow{2}{*}{$0.010^{*}$} \\
\hline & 12 & 8 & $<16.5$ & & & & & & & \\
\hline \multicolumn{4}{|c|}{$P$ value } & $\begin{array}{c}0.015^{\mathrm{a} *} \\
<0.001^{\mathrm{b} *} \\
<0.001^{\mathrm{c} *} \\
<0.001^{\mathrm{d} *} \\
0.019^{\mathrm{e}} * \\
0.286^{\mathrm{f}}\end{array}$ & $\begin{array}{c}0.004^{\mathrm{a}} * \\
0.010^{\mathrm{b}} * \\
1.000^{\mathrm{c}} \\
<0.001^{\mathrm{d} *} \\
0.004^{\mathrm{e}} * \\
0.010^{\mathrm{f}} *\end{array}$ & $\begin{array}{c}0.017^{\mathrm{a} *} \\
0.001^{\mathrm{b} *} \\
0.411^{\mathrm{c}} \\
<0.001^{\mathrm{d} *} \\
0.002^{\mathrm{e}} * \\
0.016^{\mathrm{f}} *\end{array}$ & $\begin{array}{c}0.036^{\mathrm{a} *} \\
<0.001^{\mathrm{b} *} \\
<0.001^{\mathrm{c} *} \\
0.010^{\mathrm{d} *} \\
0.048^{\mathrm{e} *} \\
0.198^{\mathrm{f}}\end{array}$ & $\begin{array}{c}0.622^{\mathrm{a}} \\
<0.001^{\mathrm{b} *} \\
0.005^{\mathrm{c} *} \\
<0.001^{\mathrm{d} *} \\
0.021^{\mathrm{e}} * \\
0.109^{\mathrm{f}}\end{array}$ & $\begin{array}{c}0.465^{\mathrm{a}} \\
<0.001^{\mathrm{b} *} \\
0.087^{\mathrm{c}} \\
<0.001^{\mathrm{d} *} \\
<0.001^{\mathrm{e}} * \\
<0.001^{\mathrm{f}} *\end{array}$ & \\
\hline
\end{tabular}

PCT, procalcitonin, CRP, C-reactive protein; APACHE II score, acute physiology and chronic health evaluation II score; PPV, Positive predictive value; NPV, Negative predictive value; AUC, area under the curve; 95\% CI, 95\% confidence interval. a, pesepsin vs. PCT; b, pesepsin vs. CRP; c, pesepsin vs. APACHE II score; d, PCT vs. CRP; e, PCT vs. APACHE II score; f, CRP vs. APACHE II score. * indicates a statistically significant correlation.

\section{Discussion}

Sepsis is one of the main challenges in emergency medicine and remains one of the most significant causes of mortality in intensive care units. Delay in the initiation of treatment is associated with worse outcomes and early diagnosis could contribute to prompt identification of those patients affected by sepsis who could benefit from quick and appropriate therapy ${ }^{[12]}$. Therefore, there is a growing interest for infection biomarkers because of the polymorphism of septic state presentations and the lack of accuracy of available biological tools ${ }^{[13]}$. Blood cultures to detect bloodstream infections are the mainstay of such attempts when patients do not display localizing signs or symptoms. 


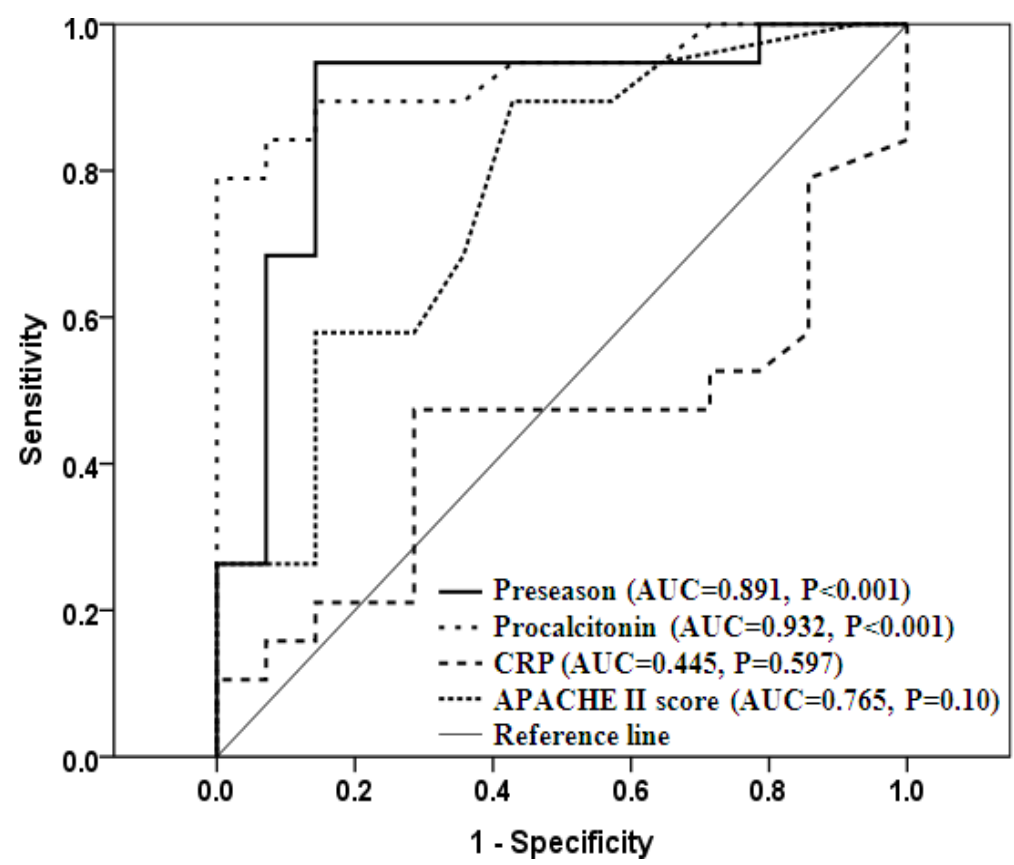

Fig. 2: Receiver operating characteristic (ROC) curves of presepsin, PCT, CRP and APACHE II score for predicting 28-day mortality in patients with sepsis.

The presence of SIRS has been shown to increase the likelihood that the blood culture will be positive but blood cultures are often negative in patients with clinical sepsis ${ }^{[14]}$.

CRP is one of the acute-phase inflammation proteins which is synthesized by the liver in response to interleukin-6 stimulation ${ }^{[15]}$. The main advantage of CRP is its great sensitivity as soon as an inflammatory process affects the human body, but unfortunately it is weakly specific for bacterial infections. Indeed, CRP levels rise in most pathological cases associated with inflammation. CRP is therefore a biomarker of inflammation rather than of infection ${ }^{[16]}$.

PCT is the pro-hormone of calcitonin that is synthesized by thyroid cells. In response to bacterial toxins, many tissues and immune cells release PCT due to a complex network with cytokines, leading to elevated serum PCT levels ${ }^{[17]}$. Although PCT has been shown to correlate closely with infection, it has some limitations, as it rises transiently in patients with non-septic conditions and SIRS and is not detectable in certain cases of sepsis ${ }^{[18]}$. CD14 is a glycoprotein expressed on the membrane surface of monocytes and macrophages and serves as a receptor for LPSs and LBPs. By activating a proinflammatory signaling cascade on contact with infectious agents, CD14 serves as a recognition molecule in the innate immune response against microorganisms. During inflammation, plasma protease activity generates SCD14 fragments. One of them, called SCD14-ST, or presepsin, is normally present in very low concentrations in the serum of healthy individuals and has been shown to be increased in response to bacterial infections. Among several molecules which have been suggested as sepsis biomarkers in recent years, presepsin, appears quite promising on the basis of its reported correlation with the early stages of the septic process ${ }^{[19]}$.

The present study aimed to investigate the diagnostic and prognostic value of presepsin in comparison to PCT and CRP in sepsis. We found that presepsin levels on admission were significantly higher in SIRS patients when compared with healthy controls. Likewise, presepsin levels on admission were significantly higher in septic patients than in non-septic patients. In accordance with our results, it has been previously reported that the concentration of presepsin was increased in patients with sepsis compared to healthy individuals and patients with SIRS ${ }^{[20-22]}$. In line with these findings, Endo et al. ${ }^{[19]}$ found that presepsin levels in patients with systemic and localized bacterial infections were significantly higher than those in patients with non-bacterial infectious diseases. The exact mechanisms of presepsin release during sepsis, and its biological function are still largely unknown ${ }^{[23]}$. One possibility is that presepsin may reflect sepsis-induced immune host defense to microorganisms. At the same time, it is worth noting that two of the strongest determinants of higher circulating presepsin concentration were renal and hepatic dysfunctions, suggesting that impaired plasma clearance may also contribute to the elevated levels of the presepsin. In agreement with a previous report ${ }^{[24]}$, we found that PCT and CRP levels on admission were significantly higher in SIRS patients than in healthy controls. Similarly, PCT levels on admission were significantly higher in septic group than in non-septic group. On the other hand, CRP levels on admission did not differ significantly between septic and non-septic groups. 
Today, there is very few data on presepsin kinetics, on how long presepsin levels are sustained, and whether presepsin levels fluctuate during the time course of sepsis. In an attempt to evaluate the usefulness of presepsin, PCT and CRP in monitoring clinical response to therapy, we measured these markers on admission, and at $1,3,5,7$ and 15 days later. In patients with sepsis, presepsin displayed the highest concentration on day 3 and tended to decrease on day 7, whereas PCT and CRP displayed the highest concentration on day 7 and tended to decrease on day 15 , suggesting that presepsin revealed earlier concentration changes when compared to PCT and CRP. Septic patients with decreasing levels of presepsin on day 7 were more likely to show improvements in clinical status leading to resolution of infection. This early decreasing trend of presepsin could be related to early clearance or to reduced production as a consequence of the appropriate treatment in the initial hours, affecting the systemic and unregulated immune response of the organism during sepsis. This observation might further enhance the diagnostic importance of presepsin and suggests that measuring presepsin may help not only in the diagnosis of sepsis, but also in monitoring the efficacy of the treatment employed. Hence, early assessment of presepsin may help in identifying, among the most severe and fatal manifestations of sepsis, those states that are still reversible, facilitating a more effective second-line and highly intensive clinical treatments. Previously, it has been described that presepsin levels begin to increase earlier than PCT and CRP, and remain elevated during 7 days before decreasing in patients with SIRS ${ }^{\text {[21] }}$.

In the current study, in all serial measurements, presepsin level was positively correlated with PCT and CRP levels. A correlation between presepsin and inflammatory biomarkers, such as CRP and PCT, can be explained by the ongoing systemic activation of inflammatory biomarkers during severe sepsis and septic shock ${ }^{[25,26]}$.

In the present study, ROC curve analysis revealed that the value of presepsin to diagnose sepsis was comparable to that of PCT, whereas presepsin demonstrated a higher diagnostic performance when compared with CRP. On the other hand, PCT tend to perform better than CRP in differentiating between septic and non-septic patients. Previous meta-analyses on the diagnostic usefulness of PCT, in comparison to CRP, reported controversial and inconsistent conclusions ${ }^{[24,27]}$. Initially, It has been demonstrated that PCT exhibited an enhanced specificity for systemic bacterial infections compared to CRP ${ }^{[28]}$, with an acceptable sensitivity depending on the site of care (emergency department or in the ICU) and the site of infectious source ${ }^{[29]}$. However, it has been subsequently demonstrated that the positive and negative predictive values of PCT are not sufficient to rule in or rule out sepsis, suggesting that PCT cannot reliably differentiate sepsis from other conditions in critically ill adult patients ${ }^{[30]}$.
Prior studies on the diagnostic accuracy of presepsin and PCT in sepsis present conflicting results. Several studies have reported that presepsin was a powerful marker, superior to PCT, for the diagnosis of sepsis in patients with a suspected infection ${ }^{[20,21,31]}$. In line with our findings, in a cohort of patients with at least one SIRS criterion, presepsin and PCT exhibited similar AUCs to differentiate patients with bacterial infection from those with non-bacterial infection ${ }^{[19]}$. In contrast, in a study by Ulla et al., the AUC of presepsin was lower than that of PCT in a cohort of patients with SIRS ${ }^{[32]}$. One explanation for these conflicting findings is the markedly lower number of bacteremic patients that were investigated in those studies. Another possibility for the inter-study variability could be related to the nature and severity of infection.

Early prediction of the outcome of patients with sepsis could be helpful in guiding therapies. Beside its contribution to the diagnosis, high PCT levels have been associated with increased mortality rates and correlated with severity scores ${ }^{[33]}$. Furthermore, it has been demonstrated that presepsin has the capacity to predict mortality and initial presepsin levels were significantly correlated with in-hospital mortality of patients affected by sepsis, severe sepsis, or septic shock ${ }^{[34]}$. The performance of presepsin to determine risk of death was found to be close to that of PCT and vary between studies ${ }^{[31,32]}$.

In the present study, in septic patients, the levels of presepsin and PCT on admission were significantly higher in non-survivors than in survivors at 28 days' follow-up, suggesting that the higher the on admission presepsin/PCT level, the more adverse the outcome in septic patients. This observation indicates that presepsin/PCT concentrations on admission are significantly correlated with 28-day in-hospital mortality in patients with sepsis. Previously, it has been reported that presepsin determined at the emergency department was significantly higher in septic non-survivors (60-day mortality) compared to survivors ${ }^{[32]}$. In the current study, the AUC of presepsin for predicting 28-day mortality in septic patients was slightly lower than that of PCT but slightly higher than that of APACHE II score. On the other hand, presepsin and PCT performed significantly better than CRP in predicting 28-day mortality. We have demonstrated that presepsin and PCT displayed comparable performance in predicting 28-day mortality in septic patients. The close correlation between presepsin/PCT initial levels and in-hospital mortality suggests that these biomarkers could be used to perform an early and reliable risk stratification and to identify high-risk patients who would rapidly benefit from prompt antibiotic therapy and other supportive treatment. Presepsin has been previously described as a powerful prognostic biomarker compared to PCT and APACHE II score for short-term 28-day all-cause mortality ${ }^{[19,31]}$. In addition, Ulla et al. ${ }^{[32]}$ demonstrated increased risk of death within 60 days in patients with increased presepsin levels $\geq 1,000 \mathrm{pg} / \mathrm{ml}$. Furthermore, in 
a retrospective analysis including patients with severe sepsis and septic shock, Masson et al. ${ }^{[34]}$ demonstrated that increased presepsin levels revealed significant prognostic value for both 28-day and 90-day all-cause mortality, whereas PCT failed to provide any prognostic information, speculating that decreasing presepsin levels in surviving patients might indicate a beneficial effect of ICU treatment.

\section{Conclusions}

In conclusion, the present study suggests that presepsin and PCT are valuable biomarkers for early sepsis diagnosis, risk stratification and predicting short-term mortality. Furthermore, our findings demonstrate that presepsin has proven to be a promising candidate for accurate and early prediction of abnormal host response, giving preliminary indications that presepsin has an unquestionable effectiveness in monitoring clinical responses to therapeutic interventions. Further studies are warranted to refine the respective contribution of presepsin as a biomarker of sepsis. Our observations are encouraging and should prompt further validation of presepsin in different clinical settings.

\section{References}

1) Bone, R. C., Balk, R. A., Cerra, F. B., Dellinger, R. P., Fein, A. M., Knaus, W. A., Schein, R. M. and Sibbald, W. J. (1992). Definitions for sepsis and organ failure and guidelines for the use of innovative therapies in sepsis. The ACCP/SCCM Consensus Conference Committee. American College of Chest Physicians/Society of Critical Care Medicine. Chest 101(6):1644-1655.

2) Annane, D., Aegerter, P., Jars-Guincestre, M. C. and Guidet, B. CUB-Réa Network. (2003). Current epidemiology of septic shock: the CUB-Réa Network. Am. J. Respir. Crit. Care Med. 168(2): $165-172$.

3) Riedemann, N. C., Guo, R. F. and Ward, P. A. (2003). Novel strategies for the treatment of sepsis. Nat. Med. 9:517-524.

4) Groselj-Grenc, M., Ihan, A., Pavcnik-Arnol, M., Kopitar, A. N., Gmeiner-Stopar, T. and Derganc, M. (2009). Neutrophil and monocyte CD64 indexes, lipopolysaccharide-binding protein, procalcitonin and C-reactive protein in sepsis of critically ill neonates and children. Intensive Care Med. 35(11):1950-1958.

5) Herzum, I. and Renz, H. (2008). Inflammatory markers in SIRS, sepsis and septic shock. Curr. Med. Chem. 15(6):581-587.

6) Reinhart, K., Meisner, M. and Brinkhorst, F. M. (2006). Markers for sepsis diagnosis: What is useful? Crit. Care Clin. 22(3):503-519.

7) Endo, S., Aikawa, N., Fujishima, S., Sekine, I., Kogawa, K., Yamamoto, Y., Kushimoto, S., Yukioka, H., Kato, N., Totsuka, K., Kikuchi, K., Ikeda, T., Ikeda, K., Yamada, H., Harada, K. and Satomura, S. (2008). Usefulness of procalcitonin serum level for the discrimination of severe sepsis from sepsis: a multicenter prospective study. $J$. Infect. Chemother. 14(3):244-249.

8) Bas, S., Gauthier, B. R., Spenato, U., Stingelin, S. and Gabay, C. (2004). CD14 is an acute-phase protein. J. Immunol. 172(7):4470-4479.

9) Shirakawa, K., Naitou, K., Hirose, J., Takahashi, T. and Furusako, S. (2011). Presepsin (sCD14-ST): Development and evaluation of one-step ELISA with a new standard that is similar to the form of presepsin in septic patients. Clin. Chem. Lab. Med. 49(5):937-939.

10) Knaus, W. A., Draper, E. A., Wagner, D. P. and Zimmerman, J. E. (1985). APACHE II: a severity of disease classification system. Crit. Care Med. 13(10):818-829.

11) Okamura, Y. and Yokoi, H. (2011). Development of a point-of-care assay system for measurement of presepsin (sCD14-ST). Clin. Chim. Acta 412(2324):2157-2161.

12) Dellinger, R. P., Levy, M. M., Rhodes, A., Annane, D., Gerlach, H., Opal, S. M., Sevransky, J. E., Sprung, C. L., Douglas, I. S., Jaeschke, R., Osborn, T. M., Nunnally, M. E., Townsend, S. R., Reinhart, K., Kleinpell, R. M., Angus, D. C., Deutschman, C. S., Machado, F. R., Rubenfeld, G. D., Webb, S. A., Beale, R. J., Vincent, J. L. and Moreno, R.; Surviving Sepsis Campaign Guidelines Committee including the Pediatric Subgroup (2013). Surviving sepsis campaign: international guidelines for management of severe sepsis and septic shock: Crit. Care Med. 41(2):580-637.

13) Rivers, E. P. and Ahrens, T. (2008). Improving outcomes for severe sepsis and septic shock: tools for early identification of at-risk patients and treatment protocol implementation. Crit. Care Clin. 24(3 Suppl):S1-S47.

14) Jones, G. R. and Lowes, J. A. (1996). The systemic inflammatory response syndrome as a predictor of bacteraemia and outcome from sepsis. QJM 89(7):515-522.

15) Póvoa, P. (2002). C-reactive protein: a valuable marker of sepsis. Intensive Care Med. 28(3):235243.

16) Clyne, B. and Olshaker, J. S. (1999). The Creactive protein. J. Emerg. Med. 17(6):1019-1025.

17) Matera, G., Quirino, A., Giancotti, A., Pulicari, M. C., Rametti, L., Rodríguez, M. L., Liberto, M. C. and Focà, A. (2012). Procalcitonin neutralizes bacterial LPS and reduces LPS-induced cytokine release in human peripheral blood mononuclear cells. BMC Microbiol. 12:68.

18) Wacker, C., Prkno, A., Brunkhorst, F. M. and Schlattmann, P. (2013). Procalcitonin as a diagnosticmarker for sepsis: a systematic review and meta-analysis. Lancet Infect. Dis. 13(5):426-435.

19) Endo, S., Suzuki, Y., Takahashi, G., Shozushima, T., Ishikura, H., Murai, A., Nishida, T., Irie, Y., Miura, M., Iguchi, H., Fukui, Y., Tanaka, K., 
Nojima, T. and Okamura, Y. (2012). Usefulness of presepsin in the diagnosis of sepsis in a multicenter prospective study. J. Infect. Chemother. 18(6):891897.

20) Yaegashi, Y., Shirakawa, K., Sato, N., Suzuki, Y., Kojika, M., Imai, S., Takahashi, G., Miyata, M., Furusako, S. and Endo, S. (2005). Evaluation of a newly identified soluble CD14 subtype as a marker for sepsis. J. Infect. Chemother. 11(5):234-238.

21) Shozushima, T., Takahashi, G., Matsumoto, N., Kojika, M., Okamura, Y. and Endo, S. (2011). Usefulness of presepsin (sCD14-ST) measurements as a marker for the diagnosis and severity of sepsis that satisfied diagnostic criteria of systemic inflammatory response syndrome. J. Infect. Chemother. 17(6):764-769.

22) Romualdo, L. G., Torrella, P. E., González, M. V., Sánchez, R. J., Holgado, A. H., Freire, A. O., Acebes, S. R. and Otón. M. D. (2014). Diagnostic accuracy of presepsin (soluble CD14 subtype) for prediction of bacteremia in patients with systemic inflammatory response syndrome in the Emergency Department. Clin. Biochem. 47(7-8):505-508.

23) Glück, T., Silver, J., Epstein, M., Cao, P., Farber, B. and Goyert, S. M. (2001). Parameters influencing membrane CD14 expression and soluble CD14 levels in sepsis. Eur. J. Med. Res. 6(8):351358.

24) Simon, L., Gauvin, F., Amre, D. K., Saint-Louis, P. and Lacroix, J. (2004). Serum procalcitonin and C-reactive protein levels as markers of bacterial infection: a systematic review and meta-analysis. Clin. Infect. Dis. 39(2):206-217.

25) Angus, D. C. and van der Poll, T. (2013). Severe sepsis and septic shock. N. Engl. J. Med. 369:840851.

26) Henriquez-Camacho, C. and Losa, J. (2014). Biomarkers for Sepsis. BioMed. Res. Int. 2014, 547818.
27) Becker, K. L., Snider, R. and Nylen, E. S. (2008). Procalcitonin assay in systemic inflammation, infection, and sepsis: clinical utility and limitations. Crit. Care Med. 36(3):941-952.

28) Assicot, M., Gendrel, D., Carsin, H., Raymond, J., Guilbaud, J. and Bohuon, C. (1993). High serum procalcitonin concentrations in patients with sepsis and infection. Lancet 341(8844):515-518.

29) Tang, B. M., Eslick, G. D., Craig, J. C. and McLean, A. S. (2007). Accuracy of procalcitonin for sepsis diagnosis in critically ill patients: systematic review and meta-analysis. Lancet Infect. Dis. 7(3):210-217.

30) Venkataraman, R. and Kellum, J. A. (2013). Sepsis: update in the management. Adv. Chronic Kidney Dis. 20(1):6-13.

31) Liu, B., Chen. Y. X., Yin, Q., Zhao, Y. Z. and Li, C. S. (2013). Diagnostic value and prognostic evaluation of Presepsin for sepsis in an emergency department. Crit. Care 17(5):R244.

32) Ulla, M., Pizzolato, E., Lucchiari, M., Loiacono, M., Soardo, F., Forno, D., Morello, F., Lupia, E., Moiraghi, C., Mengozzi, G. and Battista, S. (2013). Diagnostic and prognostic value of presepsin in the management of presepsin the emergency department: a multicenter prospective study. Crit. Care 17(4):R168.

33) Vincent, J. L. and Beumier, M. (2013). Diagnostic and prognostic markers in sepsis. Expert Rev. Anti Infect. Ther. 11(3):265-275.

34) Masson, S., Caironi, P., Spanuth, E., Thomae, R., Panigada, M., Sangiorgi, G., Fumagalli, R., Mauri, T., Isgrò, S., Fanizza, C., Romero, M., Tognoni, G., Latini, R. and Gattinoni, L.; ALBIOS Study Investigators. (2014). Presepsin, (soluble CD14 subtype) and procalcitonin levels for mortality prediction in sepsis: data from the Albumin Italian Outcome Sepsis trial. Crit. Care 18(1):R6. 